\title{
A Case of p-ANCA-Positive Propylthiouracil-Induced Pyoderma Gangrenosum
}

\author{
Jong Won Seo, M.D., Hyun Ho Son, M.D., Joon Hee Choi, M.D. ${ }^{1}$, Sook Kyung Lee, M.D. \\ Department of Dermatology, Maryknoll Medical Center, ${ }^{1}$ College of Medicine, Inje University, Busan, Korea
}

Propylthiouracil is a common medication used in patients with hyperthyroidism; it can cause perinuclear antineutrophil cytoplasmic antibodies ( $p$-ANCA) in some patients with Graves' disease. This antibody has been associated with various forms of vasculitis and neutrophilic dermatosis. Herein, we report a patient who presented with cutaneous manifestations of pyoderma gangrenosum with simultaneous development of $p$-ANCAs during PTU therapy for Graves' disease. (Ann Dermatol 22(1) $48 \sim 50,2010$ )

\author{
-Keywords- \\ p-ANCA, Propylthiouracil, Pyoderma gangrenosum
}

\section{INTRODUCTION}

Propylthiouracil (PTU), is a drug commonly used for the treatment of hyperthyroidism; it is associated with the development of antineutrophil cytoplasmic antibodies (ANCA) in 20\% of the patients treated for Graves' disease $^{1,2}$. ANCAs are found in the blood of many patients with necrotizing systemic vasculitis, and in some patients with drug-induced vasculitis and neutrophilic dermatosis such as sweet's syndrome, Behcet's disease and pyoderma gangrenosum (PG). PTU-induced PG associated with ANCAs was first described by Darben et al. ${ }^{1}$ in 1999. Several reports have described the ANCA phenomenon in patients with PG or a neutrophilic dermatosis with features of $\mathrm{PG}^{1-4}$.

In this paper, we report a case of PG associated with

Received May 23, 2009, Revised June 22, 2009, Accepted for publication June 22, 2009

Reprint request to: Sook Kyung Lee, M.D., Department of Dermatology, Maryknoll General Hospital, 12-4, Daecheong-dong, Jung-gu, Busan 600-730, Korea. Tel: 82-51-461-2564, Fax: 82-51-467-3232, E-mail: drj715@naver.com
ANCAs that was associated with PTU treatment for Graves' disease.

\section{CASE REPORT}

A 60-year-old woman presented with a painful $20 \times 10 \mathrm{~cm}$ necrotic ulcer with a boggy border on the right flank (Fig. 1A). This lesion had started as a small papule 1 month ago, developed into a pustule, and then an ulcer with blood, pus and granulation tissue, which was rapidly spreading into the adjacent normal skin. No history of trauma was noted. The patient had been receiving PTU (150 mg/day) for six years to treat Graves' disease. There were no abnormal findings on the complete blood count, serum electrolytes, liver function tests, rheumatoid factor, antinuclear antibody, renal function tests, thyroid function tests, pulmonary function tests and chest X-ray. The bacterial, fungal, and mycobacterial cultures from the ulcer were negative. Urine analysis revealed RBC casts ( 1 $\sim 4$ /whole field) and dysmorphic RBCs (83\%). The skin biopsy from the border of the ulcer demonstrated dense inflammatory infiltrates throughout the dermis and subcutis (Fig. 2A). There were perivascular mixed cell infiltrates with endothelial swelling and thrombus formation in the papillary dermis (Fig. 2B), and numerous neutrophilic and eosinophilic infiltrates with collagen degeneration in the reticular dermis (Fig. 2C). PG was suspected and the patient was started on treatment with $40 \mathrm{mg}$ of prednisolone daily. Dapsone, colchicine and cyclosporine were added for one month. However, despite multiple systemic medications, healing of the wound was not achieved. The ANCA testing was positive with a perinuclear pattern of staining ( $p-A N C A)$ : the antimyeloperoxidase antibody titer was $48 \mathrm{U} / \mathrm{ml}$ (reference range, $<20 \mathrm{U} / \mathrm{ml}$ ). The PTU was likely associated with occurrence of p-ANCAs and the development of PG in this patient. Therefore, the PTU was discontinued, and 

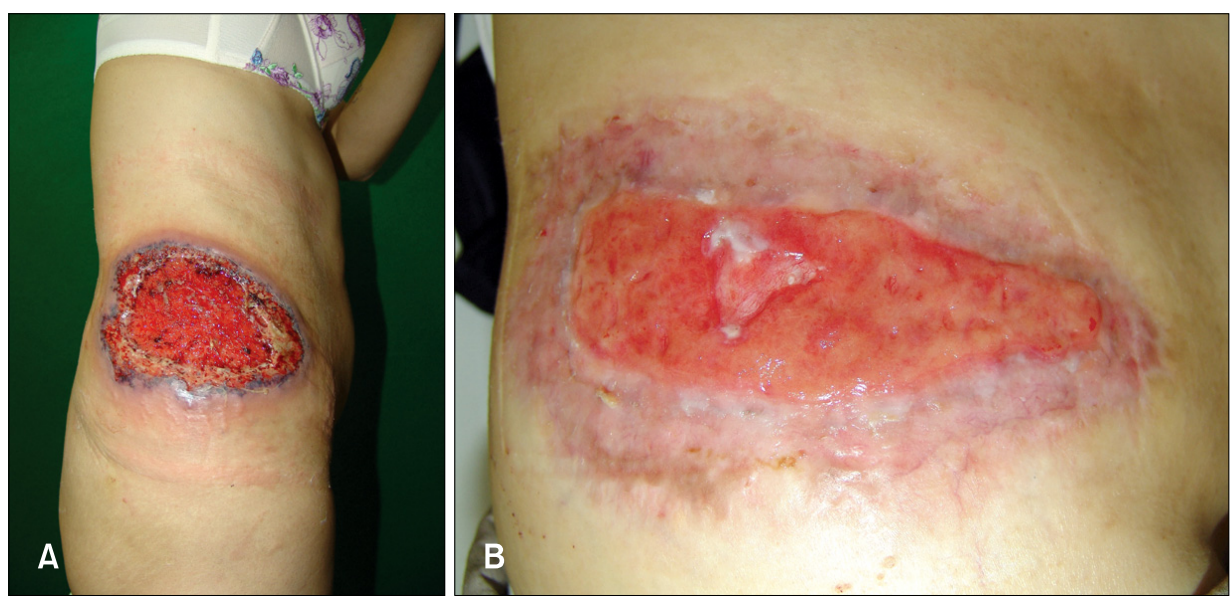

Fig. 1. (A) Initial presentation, $20 \times 10 \mathrm{~cm}$ solitary ulcer on the right flank had a violaceous border. (B) Six months after discontinuation of PTU, marked healing of the wound was observed.
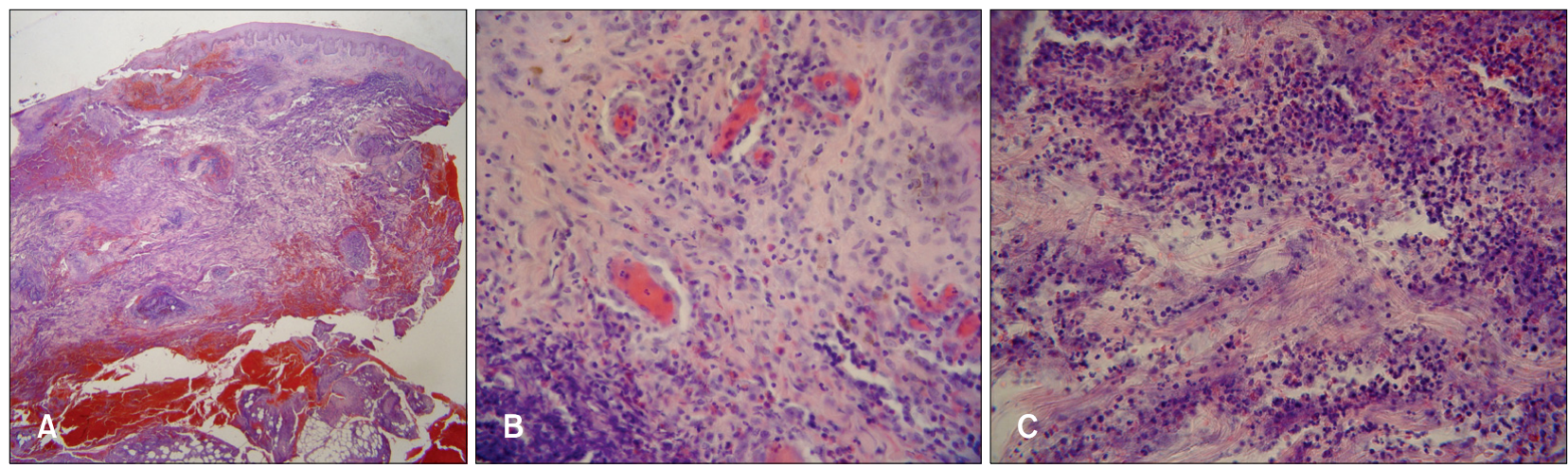

Fig. 2. (A) There are dense inflammatory infiltrates throughout the dermis and subcutis $(H \& E, \times 20)$. (B) Perivascular mixed cell infiltrates with some vascular damage in the papillary dermis $(\mathrm{H} \& \mathrm{E}, \times 400)$. (C) Dense neutrophilic and eosinophilic infiltrates with leukocytoclasia and collagen degeneration in the reticular dermis $(\mathrm{H} \& \mathrm{E}, \times 400)$.

subsequent healing of the wound was observed (Fig. 1B). After drug withdrawal, the microscopic hematuria resolved over two months and indirect immunofluorescence testing for ANCAs was negative over the next nine months.

\section{DISCUSSION}

Pyoderma gangrenosum (PG) is a rare inflammatory disorder of unknown etiology characterized by neutrophilic infiltration of the dermis and destruction of tissue. Over fifty percent of patients with PG have an underlying systemic disease, most commonly inflammatory bowel disease, IgA monoclonal gammopathy, rheumatoid arthritis, Behcet's disease, or myeloproliferative disorder ${ }^{1,2}$. Drugs causing PG have been rarely reported and include iodide, bromide, isotretinoin, granulocyte colony stimulating factor and granulocyte-macrophage colony stimulating factor ${ }^{1,3,4}$. Notably the association of PG-like ulceration with positive ANCAs after PTU therapy was first described by Darben et al. ${ }^{1}$ in 1999. Several additional studies have also reported the occurrence of PG associated with PTU therapy ${ }^{2-4}$. According to our review of the literature ${ }^{1-4}$, ANCA-positive PTU-induced PG usually affects women and appears two to three years after taking the medication for Graves' disease. Symptoms such as fever, fatigue, arthralgia and skin ulceration have been reported. Indirect immunofluorescent testing for ANCAs reveals a perinuclear pattern in all reported cases. ANCAs may persist for more than one year despite the resolution of clinical features.

PTU is associated with systemic complications such as agranulocytosis, hepatotoxicity and drug-induced hypersensitivity in $1 \%$ to $5 \%$ of patients taking this drug ${ }^{5}$. The incidence of ANCAs in patients treated with PTU varies from $20 \%$ to $67 \%$ according to published reports ${ }^{2,6}$. Among the ANCA-positive patients on PTU therapy, only $10 \%$ to $20 \%$ of patients develop vasculitis. High doses of PTU therapy, the levels of antithyroglobulin antibody and severity of Graves' disease may be associated with p- 
ANCA positivity $^{6}$. ANCA testing is now part of the clinical work-up of patients with systemic vasculitis. ANCAs have also been identified in patients with neutrophilic dermatosis due to the pathogenic role of ANCAs in the activation of neutrophils ${ }^{2-4}$.

ANCAs are autoantibodies directed against azurophilic protein in neutrophils ${ }^{3}$. The ANCAs (c-ANCA) in the cytoplasm reflect the presence of anti-serine protease-3 antibodies (PR3) and tends to be observed with Wegener's granulomatosis; whereas perinuclear ANCAs (p-ANCA) generally reflect the presence of antimyeloperoxidase antibodies and tends to be associated with microscopic polyangiitis, Churg-Strauss disease and pauci-immune crescent glomerulonephritis ${ }^{7}$. p-ANCAs also have been associated with drug-induced vasculitis secondary to agents such as PTU, hydralazine, carbimazole, minocycline and penicillamine ${ }^{1,8,9}$.

The role of PTU in the pathogenesis of PG is unknown. However, it has been suggested that PTU selectively accumulates in the neutrophils and binds to myeloperoxidase (MPO), resulting in a change of the heme structure of the enzyme. The altered enzymes, possibly combined with the drug, might act as haptens and initiate production of anti-MPO autoantibodies, which then stimulate other neutrophils ${ }^{10}$. Activated primed neutrophils degranulate and produce oxygen free radicals, increasing the expression of adhesion molecules on both neutrophils and endothelial cells, stimulating the release of interleukin 8 and interfering with control of the enzyme activity of these target antigens $3,5,10$

The present case was typical of a PTU-induced PG in terms of gender, cutaneous manifestations and the $p$ ANCA pattern; however, the duration of treatment before the onset of complications, differed from the timing reported by Darben et al. ${ }^{1}$. The clinical improvement and concomitant lowering of the antibody titers after discontinuing the PTU implicate that the drug in the production of this autoantibody and the pathogenesis of this disorder.

In conclusion, p-ANCA-positive PTU-induced PG is a rare side effect of this drug. However, physicians should be aware of this complication during PTU therapy; recogni- tion of the skin lesion and identification of p-ANCAs permits early diagnosis and alteration of treatment. Further studies are needed to clarify the role of PTU in the development of ANCAs and the pathogenesis of PG.

\section{REFERENCES}

1. Darben T, Savige J, Prentice R, Paspaliaris B, Chick J. Pyoderma gangrenosum with secondary pyarthrosis following propylthiouracil. Australas J Dermatol 1999;40:144146.

2. Miller RM, Darben TA, Nedwich J, Savige J. Propylthiouracil-induced antineutrophil cytoplasmic antibodies in a patient with Graves' disease and a neutrophilic dermatosis. Br J Dermatol 1999;141:943-944.

3. Hong SB, Lee MH. A case of propylthiouracil-induced pyoderma gangrenosum associated with antineutrophil cytoplasmic antibody. Dermatology 2004;208:339-341.

4. Gungor K, Gonen S, Kisakol G, Dikbas O, Kaya A. ANCA positive propylthiouracil induced pyoderma gangrenosum. J Endocrinol Invest 2006;29:575-576.

5. Jacobs EM, Hartkamp A, Kaasjager HA. PTU-associated cutaneous vasculitis with ANCA anti-MPO and anti-PR3 antibodies. Neth J Med 2003;61:296-299.

6. Sato $H$, Hattori $M$, Fujieda $M$, Sugihara S, Inomata $H$, Hoshi $M$, et al. High prevalence of antineutrophil cytoplasmic antibody positivity in childhood onset Graves' disease treated with propylthiouracil. J Clin Endocrinol Metab 2000;85: 4270-4273.

7. Kitahara T, Hiromura K, Maezawa A, Ono K, Narabara N, Yano $\mathrm{S}$, et al. Case of propylthiouracil-induced vasculitis associated with anti-neutrophil cytoplasmic antibody (ANCA); review of literature. Clin Nephrol 1997;47:336-340.

8. Miller RM, Savige J, Nassis L, Cominos BI. Antineutrophil cytoplasmic antibody (ANCA)-positive cutaneous leucocytoclastic vasculitis associated with antithyroid therapy in Graves' disease. Australas J Dermatol 1998;39:96-99.

9. Wang LH, Tsai MJ, Tsai WY, Lee JS, Chiang BL. Propylthiouracil-induced antineutrophil cytoplasm antibodypositive anaphylactoid purpura-like vasculitis--a case report. J Formos Med Assoc 2000;99:642-645.

10. Sheen YS, Chu CY, Yu HS. Antineutrophil cytoplasmic antibody-positive cutaneous leukocytoclastic vasculitis associated with propylthiouracil therapy. Arch Dermatol 2006; 142:879-880. 